\title{
PROCALCITONIN AS A MARKER FOR EARLY DIAGNOSIS OF SEPSIS
}

\author{
${ }^{1}$ Rahim Raoofi, ${ }^{2}$ Zahra Salmani, ${ }^{2}$ Fatemh Moradi, \\ ${ }^{3}$ Abdolrerza Sotoodeh and ${ }^{3}$ Saeed Sobhanian \\ ${ }^{1}$ Department of Inernal Medicine, \\ ${ }^{2}$ Student Research Committee, \\ ${ }^{3}$ Department of Immunology, \\ Faculty of Medicine, Jahrom University of Medical Sciences, Iran
}

Received 2013-11-04, Revised 2013-11-21; Accepted 2013-12-22

\begin{abstract}
Sepsis is a critical condition often caused by bacterial infection and associated with death and mortality. The prognosis of this disease depends on early diagnosis and proper treatment. Definite diagnosis of sepsis is positive blood culture and this test needs a long time to perform, so other biochemical parameters such as procalcitonin serum level has been introduced. To determine sensitivity, specificity, positive and negative predictive value of procalcitonin serum level at first time and $72 \mathrm{~h}$ after admission and to compare it with blood culture test for diagnosis of sepsis. Blood sample for blood culture, prepheral blood smear at first time and procalcitonin serum level measuring by semi quantitative method at first and $72 \mathrm{~h}$ after admission were sampled. Nine patients had positive blood cultures. Peripheral blood smear was positive in 38 patients, (29 patients: gram stain positive, 6 patients: gram stain negative and 3 patients: both gram positive and gram negative). At first time $76.8 \%$ had positive procalcitonin $\left(>0.5 \mathrm{ng} \mathrm{mL}^{-1}\right)$ and after $72 \mathrm{~h} 65 \%$ of patients had positive procalcitonin. Sensitivity, specificity, positive and negative predictive value of procalcitonin at the first time were 100, 16,16 and $100 \%$ and for procalcitonin after $72 \mathrm{~h}$ respectively were $75,35,15$ and $90 \%$. This study showed that sensitivity of procalcitonin serum level can be used for diagnosis of sepsis. Procalcitonin increased as severity of sepsis and this study suggests high serum level of procalcitonin after $72 \mathrm{~h}$ might indicate poor outcome.
\end{abstract}

Keywords: Sepsis, Procalcitonin, Sensitivity, Specificity

\section{INTRODUCTION}

Sepsis is one of the main problems in most of health centers and it is life-threatening disease that causes wide spread mortality and morbidity in the world. Indeed, sepsis is the result of a reaction between infectious microorganisms and the host immune system. Rapid diagnosis in its early stages and treatment by appropriate antibiotics leads to reduce patients' mortality (Mandell et al., 1995).

To define the word, "sepsis" has a complete association with SIRS concept (systemic inflammatory response syndrome). SIRS is the body's inflammatory response. To define, it is when the following criteria are available:

- Temperaturemore than $38^{\circ} \mathrm{C}$ or less than $36^{\circ} \mathrm{C}$

- Heartbeat rate more than 90 beats per minute

- Respiratory rate more than 20 times per minute, or paco 2 less than $32 \mathrm{mmHg}$

- White blood cell count more than 12,000 or less than 4000, or bandemia more than $10 \%$ (Gidela et al., 2008; Mandell et al., 1995)

Corresponding Author: Abdolrerza Sotoodeh Jahromi, Department of Immunology, Faculty of Medicine, Jahrom University of Medical Sciences, Iran 
In the presence of SIRS and probable or confirmed infectious focus, the sepsis may be occurred (Gidela et al., 2008; Mandell et al., 1995). These criteria have low sensitivity and specificity for detecting disease, in spite of the fact that the existing of non-infectious various disease and conditions, including trauma, pancreatitis, cardiac infarction, cardiac tapenade and cause to make false positive and negative results in diagnosis of SIRS (Canan et al., 2003; Mandell et al., 1995). At the present, diagnosis of sepsis is a controversial. Standard laboratory tests and the clinical signs have low sensitivity and specificity, since the most severely ill patients will reveal some degree of inflammatory response, while they may have sepsis or not (Arkader et al., 2006).

Despite the fact that one of the key elements for diagnosis is blood cultures test, but it has some problems such as not being positive in the majority of cases and delay in obtaining positive results, all of which shows that an additional marker is required for diagnosis of sepsis (Kopterides and Tsangaris, 2012). In this regard, several biomarkers are being introduced. For example different types of cytokine, such as TNF-a, IL10 and CRP and also procalcitonin which are more suggested by the useful recent studies and have been able to become a head of other markers (Heper et al., 2006; Charalampos and Vincent, 2010).

Regarding to this fact that early and proper diagnosis of sepsis is one of main strategies for reducing mortality, so, the importance of diagnosis and also the lack of strong standard criteria for diagnosis has directed the last sepsis international conference, in the presence of possible or absolute infection, toward check some of biochemical and hemodynamic markers. One of these markers is Procalcitonin (a prohormones which composed of 116 amino acids) which is very useful in sepsis diagnosis in cases that the blood culture test is negative and its level is associated with sepsis severity (Canan et al., 2003). It seems that specificity of this marker is higher than CRP and WBC count and it tends to rise quickly as bacterial infections (but not viral infections) develop, increase with the severity of infection and decline with improvement (PCCM, 2013).

Few studies have been done on the distinguish value of procalcitonin serum levels and their results have been controversial (PCCM, 2013; Makoo et al., 2010; Heper et al., 2006).

Considering the importance of distinguishing sepsis, starting antibiotic therapy in and avoiding unnecessary antibiotic therapy in non-sepsis cases this study was designed to determine sensitivity and specificity of this marker in the diagnosis of sepsis.

\section{METERIALS AND METHODS}

This study was done to determine the sensitivity, specificity and predictive values of procalcitonin on diagnosis of sepsis on 60 patients 59-68 years, whit sepsis criteria, including 35 men and 25 women, who admitted to emergency unit of Peymaniye hospital of Jahrom, Iran.

After being secure about the subjects had sepsis criteria, the serum level of procalcitonin was determined. The basis of positive clinical and lab tests $0.5 \mathrm{ng} \mathrm{mL}^{-1}$ of serum pricalcitonin was cut off point and the negative and positivity levels were considered as following:

- $\quad 0.5-2 \mathrm{ng} \mathrm{mL}^{-1}$ weakly positive

- $\quad 2-10 \mathrm{ng} \mathrm{mL}^{-1}$ positive

- $10 \mathrm{ng} \mathrm{mL}^{-1}<$ strongly positive

In order to find the possible source of infection and organ dysfunction, disease details and checkup result for the patients were gathered and then serum level of procalcitonin was determined by semi quantitative immunochromatogrsaphy method by commercial kit, at the time of visiting and over a period of $72 \mathrm{~h}$.

Other necessary examinations for sepsis diagnosis such as blood culture test was done during the first 24 hours of hospitalization and other tests, such as analysis test and urine culture, chest radiography, ESR, CRP, LFT and Lp (if it was necessary) was taken simultaneously.

In addition, at the same time, when the patient sample was given for blood culture, it was used to examine the smear for microbiological assessment to examine for the presence of gram-negative and grampositive microorganisms. Finally, patients were arranged in to the following groups:

Group1: Patients with positive blood cultures

Group2: Patients with positive peripheral blood smear

Group3: Patients have negative blood culture and smear and positive inflammatory markers (in flammatory marker means ESR and CRP)

Group4: Patients with negative blood cultures and smears and also negative inflammatory markers 
According to the degree of sepsis severity, patients are placed in one of the following groups:

Group1: SIRS

Group2: Sepsis

Group3: Severe sepsis

Group4: Shock sepsis

That is, for placement in a specific group, the patient must has the characteristics of that group. In group 2, the patients have the SIRS symptoms with the possible infection source; Group3 included patients who had at least one of the following criteria which in fact reveal the organsdys function:

- $\mathrm{Pao}_{2} / \mathrm{FIO} 2<300<$ urine output $/ 05 \mathrm{~mL} / \mathrm{kg} / \mathrm{hr}$

- Serum cratenin $>2 \mathrm{mg} \mathrm{dL}^{-1}$

- $\mathrm{INR}<1 / 5$ or $\mathrm{PT}>60$

- Platelet count $<100000$

- Total bilirubin $>4 \mathrm{mg} \mathrm{dL}^{-1}$

- $\mathrm{PH}<7 / 35$ Systolic $\mathrm{Bp}<90$ OR Mean arterial $\mathrm{Bp}<60 \mathrm{OR}$ less $50 \mathrm{~mL} \mathrm{Hg}$ compare to last base blood pressure. It response to fluid therapy

Group 4 was the patients of this group whom suffered from blood pressure drop and this drop of blood pressure not response to therapeutic fluid and needs vasoconstractive agent.

In these two divisions, the PCT was measured at the time of zero and $72 \mathrm{~h}$ then the results of the procalcitonin and gold standard tests compared to each other and the sensitivity, specificity, positive and negative predictive values were calculated.

\section{RESULTS}

The widest spread infection was of the lung infection by $58.3 \%$ (35 patients) and then, the urinary tract $16.7 \%$ (10 patients), digestive $11.7 \%$ (7 patients), skin and soft tissue 6.7\% (4 patients) and infections with unknown origin $6.7 \%$ (4 patients). Of the whole study population, 9 patients were reported as positive blood culture and that was the most common microorganism was Streptococcus. The Peripheral blood smear and gram-stained for 38 patients turned out to be positive and of which 29 patients had grampositive, 6 patients had gram-negative and 3 patients had peripheral blood smear, including gram-positive and gram-negative. In this study, 5 patients died and their serum level of procalcitonin in zero times were as following:

- In 4 patients (19\%), positive (2-10 $\left.\mathrm{ng} \mathrm{mL}^{-1}\right)$

- In one patient (5\%), strongly positive (greater than $10 \mathrm{ng} \mathrm{mL}^{-1}$ )

There was not found any significant correlation between serum level of procalcitonin at times zero and $72 \mathrm{~h}$ with gender and age $(\mathrm{p}=0.113, \mathrm{p}=0.121$, respectively). Blood culture was reported as positive in $15 \%$ (9 patients). Blood culture tests were negative in all patients who had negative procalcitonin serum level at time zero.

Also, there was not found any significant correlation between Procalcitonin amount of times zero and 72 and blood culture ( $\mathrm{p}=0.119, \mathrm{p}=0.125$, respectively).

The average serum level of Procalcitonin at time zero was reported of $9.2 \mathrm{ng} \mathrm{mL}^{-1}$ in patients with positive and negative blood culture tests, but after $72 \mathrm{~h}$ the average serum level of Procalcitonin was reduced in the both groups, while the group who had negative blood cultures, this reduction was more noticeable. Procalcitonin reduction was not significant in patients with blood culture positive $(\mathrm{p}=0.070)$, but these changes showed a significant statistical correlation in patients with negative blood cultures $(\mathrm{p}=0.0001)$.

In patients with positive blood culture, based on the type of microorganism, the most percentage was pertained to streptococci organism and then Staph, E. coli and Enterococcus, respectively. No significant correlation was seen in time zero and $72 \mathrm{~h}$ type microorganisms $(p=0.360)$.

It was reported that 38 patients $(63.3 \%)$ had positive smear. The proportion of gram-positive organisms were 29 smears and gram-negative were 6 smears and 3 smears were mixture of positive and negative. So, there were no significant correlation between procalcitonin amount and peripheral blood smear in any of them $(\mathrm{P}=$ $0.417, \mathrm{p}=0.500$, respectively).

In zero times, in positive smear patients, the average of serum level of procalcitonin was non-significantly more than patients with negative smear and after passing $72 \mathrm{~h}$, it was seen that the mean serum level of procalcitonin reduced in the two groups, which in patients positive smear this reduction was more visible.

The serum level of procalcitonin changes, in positive smear and negative smear patients, were significant in the two groups $(\mathrm{p}=0.000)$. 
Among the $13.3 \%$ of the population with a negative procalcitonin serum level at zero time, $87.5 \%$ were categorized in the sepsis group and $12.5 \%$ were placed in severe sepsis group. Which there was seen no significant correlation between the procalcitonin serum level and sepsis severity at times zero and $72(\mathrm{p}=0.251, \mathrm{p}=$ 0.290 , respectively).

Though, after passing $72 \mathrm{~h}$, the procalcitonin serum level decreased in all three groups and this reduction was more visible in patients of shock sepsis group than other groups.

In sepsis and severe sepsis patients, the changes of procalcitonin serum level was significant $(\mathrm{p}=0.000)$, but this changes were not significant in those with sepsis shock $(\mathrm{p}=0.180)$.

The under studying patients were also examined on the site of infection. From the $13.3 \%$ of patients with a negative procalcitonin serum level at time zero, $62.5 \%$ had lung infection, $12.5 \%$ had urinary tract infections, $12.5 \%$ had skin and soft tissue infections and $12.5 \%$ had unknown local infection.

There was not observed a significant correlation between the procalcitonin serum level at time zero and infection place $(\mathrm{p}=0.335)$. But after passing $72 \mathrm{~h}$ and measuring the procalcitonin serum level, it was revealed that the procalcitonin serum levelin $35 \%$ of the total population was positive.

Of this $35 \%$ with a negative procalcitonin in time 72 h, $52.4 \%$ patients had pulmonary infection, $28.6 \%$ had urinary tract infection, $4.8 \%$ had digestive infection, 9.5\% had skin and soft tissue infections and $4.8 \%$ were infected with an unknown location.

There was a significant correlation between the procalcitonin serum level, at the time $72 \mathrm{~h}$ the place of infection $(\mathrm{p}=0.033)$.

This significant correlation was $58.3 \%$ for patients with lung origin, $16 / 7 \%$ for urinary tracts origin, $11.7 \%$ for digestive origin, $6.7 \%$ for skin origin and $6.7 \%$ for patients with unknown origin.

The patients were examined to find that whether they have positive or negative inflammatory markers and finally the statistically correlation was not significant.

In group of patients with negative blood culture, negative peripheral blood smear and negative inflammatory marker, the relation of different variables such as age, gender, severity of sepsis and place of infection, in times zero and 72, was not seen a significant statistically correlation except in case of gender and in time $72(\mathrm{p}=0.010)$.
In this study, $8.3 \%$ (5 patients) had died. It was reported that all patients who died have positive procalcitonin serum levelbut correlation was not significant $(\mathrm{p}=0.159)$.

The serum level of procalcitonin in time $72 \mathrm{~h}$ as compared to 0 , in patients who had died, had been fallen down and this was not significant $(\mathrm{p}=0.590)$.

The sensitivity, specificity, positive and negative predictive values for procalcitonin serum level were examined in zero and $72 \mathrm{~h}$ which its result was optained, in case of time zero, as 100, 16, 16 and $100 \%$ respectively and in case of $72 \mathrm{~h}$ it was 75,35 , 15 and $90 \%$ respectively.

\section{DISCUSSION}

Through various studies, procalcitonin was one of the markers which have been suggested for more rapid diagnosis of sepsis (Canan et al., 2003).

In our study, we examined the sensitivity, specificity, positive and negative predictive values for procalcitonin at time zero and $72 \mathrm{~h}$ which its result was reported, in case of time zero, as 100, 16, 16 and $100 \%$ respectively; and in case of $72 \mathrm{~h}$ it was 75,35 , 15 and $90 \%$ respectively (Table 1).

Other studies that have been conducted show similar sensitivity but lower specificity. Of these studies, Canan et al. (2003) have stated that the procalcitonin sensitivity and specificity for differentiating SIRS from sepsis, is 85 and $91 \%$ respectively.

Another study which was conducted by Zahra Ahmadinejad et al. (2009) presents that the sensitivity and specificity for procalcitonin $\left(0.5 \mathrm{ng} \mathrm{mL}^{-1}\right)$ are respectively, 89.2 and $82.9 \%$. Another study by Heper et al. (2006) expressed the sensitivity and specificity for procalcitonin by 88 and $70 \%$ respectively.

In a study conducted by Wanner et al. (2005), it is expressed that the procalcitonin sensitivity and specificity is low in diagnosis of sepsis. Brunkorst et al. (2000) and in a meta-analysis showed 77 and $85 \%$ for sensitivity and specificity respectively (Wacker et al., 2013).

Table 1. Sensitivity, specificity, positive and negative predictive values for PCT serum level at time zero and $72 \mathrm{~h}$ after admission

\begin{tabular}{lllll}
\hline $\begin{array}{l}\text { PCT serum } \\
\text { level }\end{array}$ & Sensitivity\% & Specificity\% & NPV\% & PPV\% \\
\hline Zero time & 100 & 16 & 100 & 16 \\
$72 \mathrm{~h}$ & 75 & 35 & 90 & 15 \\
\hline
\end{tabular}

NPV: Negative Predictive Value; PPV: Positive Predictive Value 
However, in present study there was not a significant correlation between procalcitonin serum level sat zero and $72 \mathrm{~h}$ times with gender; which these results are in agreement with other studies (Heper et al., 2006; PCCM, 2013; Makoo et al., 2010; Ahmadinejad et al., 2009). Although, in present study, in a group of patients who have a negative blood cultures, negative peripheral blood smears and negative inflammatory marker, there was a significant correlation between procalcitonin amount of 72 $\mathrm{h}$ and gender and it was $75 \%$ for female and $25 \%$ for male gender, but it should be noted that there were 8 patients in this group and most of them, 6 patients, were female.

But there was a significant correlation between procalcitonin amount, in time $72 \mathrm{~h}$ and the place of infection; which this relation was about $58.3 \%$ with lung origin and this result was in contrast to Heper results, because they did not find any significant correlation between procalcitonin serum level, in time 24, 48 and 72 $\mathrm{h}$ and place of infection (Heper et al., 2006).

In the study that we carried out most of the patients had pulmonaryorigin $(58.3 \%)$, the procalcitonin serum level, in time $72 \mathrm{~h}$ compared to time zero, had been decreased in all the population and this was significant, so it can be concluded that there was a relationship with the place of infection.

In the study we have done, in connection with the procalcitonin serum level at the times zero and $72 \mathrm{~h}$ and blood cultures, there was found no significant correlation; which it was in harmony with Heper studies (Angeletti et al., 2012). But it was in contrast to the study by Ghorbani (2009) which it was concluded that there exist a significant correlation between the procalcitonin serum level and patients with positive blood cultures.

In the present study there is not a significant correlation between the procalcitonin amount in time zero and $72 \mathrm{~h}$ and type of microorganisms. This result is in parallel with the result of a study which was done on patients with infective endocarditic, which showed a significant correlation between the procalcitonin amount and Staphylococcus aurous in the infective endocarditic factor (Cuculi et al., 2008).

In present study, it was realized that procalcitonin cannot discern between patients with different levels of sepsis (sepsis, severe sepsis and sepsis shock). Here, the findings of Ghorbani (2009) are in agreement with mentioned results, but in the study was done by Brunkorst et al. (2000) about the value of determining of PCT level in early diagnosis of SIRS patients, sepsis, severe sepsis and septic shock, compared with WBC, CRP, platelet counting and AP-IL, it revealed this result that procalcitonin can distinguish between severe sepsis and sepsis shock groups.

It is worthy to mention that in Brunkhorst study, $21 \%$ of patients were belonged to septic shock group, while in our study only $3.3 \%$ of population had septic shock (Brunkorst et al., 2000).

In present study the patients were categorized into two groups: sepsis and severe sepsis (severe sepsis and septic shock) and here it was seen that also in these patients the procalcitonin, in times zero and 72 $\mathrm{h}$, cannot make a difference between these two groups and this result is in contradiction with (Heper et al., 2006) thus, here it can be pointed that there is the probability of using antibiotics by patients before they hospitalized in hospital.

In present study the procalcitonin serum level, in the two times of zero and $72 \mathrm{~h}$, in patients who died had higher procalcitonin serum level than patients survived; that is, in this case the result is in accordance with Heper results (Heper et al., 2006).

And also in present study there was not seen any significant correlation between procalcitonin serum level changes in time zero and $72 \mathrm{~h}$ in patients who died, but it was significant for patients who survived similar result showed in the study that was done by (Ikeda et al., 2012).

The last no table point is that in patients who had precaution awareness, procalcitonin serum level changes had less reduction in time $72 \mathrm{~h}$ than time 0 it can be stated that the procalcitonin serum level highness in time $72 \mathrm{~h}$ is useful for patients with precaution awareness.

\section{CONCLUSION}

In this study it was shown that procalcitonin serum levels have a high sensitivity in sepsis diagnosis and therefore, accompanying with other patient's clinical symptoms, are a suitable marker in sepsis diagnosis.

In time $72 \mathrm{~h}$, the features of this substance had been higher than time zero, it is recommended more studies for more reliable results.

Since there is not a significant difference between the procalcitonin serum level, in time $72 \mathrm{~h}$ compared to time zero, in positive blood culture patients than negative ones and in sepsis shock patients than sepsis and severe sepsis, it can be expressed that the procalcitonin serum level decreases in a lower rate in sicker patients.

In this study the procalcitonin serum level had been decreased significantly in patients who died than whom 
they are survived, so the richness of this marker comes with terrible precaution in patients.

\section{ACKNOWLEDGMENT}

The researchers would like to thank microbiologic ward of Jahrom University of Medical Science for their kind cooperation. This article has been extracted from the result of medical student thesis to take a general physician degree from jahrom University of Medical Science by Miss Zahra Salmani and Miss Zahra Moradi.

\section{REFRENCES}

Ahmadinejad, Z., B. Dadsetan, M. Jalili, A. Soudbakhsh and M. Rasolinejad, 2009. Evaluation of serum procalcitonin in patients with systemic inflammatory response syndrome with and without infection. Acta Mdica Iranica., 47: 383-388. DOI:

Angeletti, S., F. Battistoni, M. Fioravanti, S. Bernardini and G. Dicuonzo, 2012. Procalcitonin and midregional pro-adrenomedullin test combination in sepsis diagnosis. Clin. Chem. Lab. Med., 51: 10591067. DOI: $10.1515 / \mathrm{cclm}-2012-0595$

Arkader, R., E.J. Troster, M.R. Lopes, R.R. Junior and J.R. Carcillo et al., 2006. Procalcitonin does discriminate between sepsis and systemic inflammatory response syndrome. Display Sett., 91: 117-120. DOI: 10.1136/adc.2005.077446

Brunkorst, F.M., K. Wegscheider, Z.F. Forycki, R. Brunkhorst, 2000. Procalcitonin for early diagnosis and differentiation of SIRS, sepsis, severe sepsis and septic shock. Int. Crit. Care Med., 2: 148-52. DOI: 10.1007/BF02900728

Canan, B., S. Hulya, G. Ercan, S. Ugur and K. Bunyamin, 2003. Usefulness of procalcitonin for diagnosis of sepsis in the intensive care unit. Display Sett., 7: 85-90. DOI: 10.1186/cc1843

Charalampos, P. and J.L. Vincent, 2010. Sepsis biomarkers: A review. Crit. Care, 14: R15-R15. DOI: $10.1186 / \mathrm{cc} 8872$

Cuculi, F, S. Toggweiler, M. Auer, A.D. Maur and M. Zuber et al., 2008. Serum procalcitonin has the Potential to identify staphylococcus aureusendocarditis. Eur. J. Clin. Microbiol. Infect., 27: 1145-1149. DOI: 10.1007/s10096-008-0541-3

Ghorbani, G.H., 2009. Procalcitonin role in differential diagnosis of infection stages and non infection inflammation. Pak. J. Biol. Sci., 12: 393-396. DOI: PMID: 19579976
Gidela, D., D.L.L. Rosa, M. Valencia and C.M. Arango, 2008. Toward and operativedingninsis in sepsis: A latent class approach. BMC Infect Dis., 8: 18-18.

Heper, Y., E.H. Akalın, R.S. Mistik, S. Akgoz and O. Tore, 2006. Evaluation of serum C-reactive protein, procalcitonin, tumor necrosis factor alpha and interleukin-10 levels as diagnostic and prognostic parameters in patients with community-acquired sepsis, severe sepsis and septic shock. Eur. J. Clin. Microbiol. Infect. Dis., 25: 481-491. DOI: 10.1007/s10096-006-0168-1

Ikeda, T., K. Ikeda and S. Suda, 2012. Procalcitonin level as a marker of severe sepsis and septic shock patients who required polymyxin-B immobilized fiber with direct hemoperfusion. Crit. Care, 16: 55. DOI: $10.1186 / \mathrm{cc} 11692$

Kopterides, P. and I. Tsangaris, 2012. Procalcitonin and sepsis: Recent data on diagnostic utility prognostic potential and therapeutic implications in critically ill patients. Minerva Anestesiol, 78: 823-835. PMID: 2561677

Makoo, Z.B., H.R. Soltani, A. Hasani, R.B. Makoo and O. Mashrabi, 2010. Diagnostic value of serum and serum and cerebrospinal fluid procalcitonin in differentiation bacterial from aseptic meningitis. Am. J. Infect. Dis., 6: 93-97. DOI: 10.3844/ajidsp.2010.93.97

Mandell, G.L., J.E. Bennett and R.G. Douglas, 1995. Principles and Practice of Infectious Disease. 2nd Edn., Wiley, New York, ISBN-10: 0471876437, pp: 1760 .

PCCM, 2013. Can procalcitonin help guide therapy for suspected pneumonia and other infections? PulmCCM.org.

Wacker, C., A. Prkno, F.M. Brunkhorst and P. Schlattmann, 2013. Procalcitonin as a diagnostic marker for sepsis: A systematic review and metaanalysis. Lancet Infect. Dis., 13: 426-435. DOI: 10.1016/S1473-3099(12)70323-7

Wanner, C., V. Krane, W. Marz, M. Olschewski and J.F.E. Mann, 2005. Atorvastatin in patients with type 2 diabetes mellitus undergoing hemodialysis. N. Engl. J. Med., 353: 238-248. DOI: 10.1056/NEJMoa043545 